\title{
Constraining sub-grid physics with high-redshift spatially-resolved metallicity distributions (Research Note)
}

\author{
B. K. Gibson ${ }^{1}$, K. Pilkington ${ }^{1}$, C. B. Brook ${ }^{2}$, G. S. Stinson ${ }^{3}$, and J. Bailin ${ }^{4}$ \\ 1 Jeremiah Horrocks Insitute, University of Central Lancashire, Preston, PR1 2HE, UK \\ e-mail: bkgibson@uclan.ac.uk \\ 2 Departamento de Física Teórica, Universidad Autónoma de Madrid, Cantoblanco, 28049 Madrid, Spain \\ 3 Max-Planck-Institut für Astronomie, Königstuhl 17, 69117 Heidelberg, Germany \\ ${ }^{4}$ Department of Physics \& Astronomy, University of Alabama, Tuscaloosa, AL 35487-0324, USA \\ Received 5 February 2013 / Accepted 5 April 2013
}

\begin{abstract}
Aims. We examine the role of energy feedback in shaping the distribution of metals within cosmological hydrodynamical simulations of $\mathrm{L}^{*}$ disc galaxies. While negative abundance gradients today provide a boundary condition for galaxy evolution models, in support of inside-out disc growth, empirical evidence as to whether abundance gradients steepen or flatten with time remains highly contradictory.

Methods. We made use of a suite of $\mathrm{L}^{*}$ discs, realised with and without "enhanced" feedback. All the simulations were produced using the smoothed particle hydrodynamics code Gasoline, and their in situ gas-phase metallicity gradients traced from redshift $z \sim 2$ to the present-day. Present-day age-metallicity relations and metallicity distribution functions were derived for each system.

Results. The "enhanced" feedback models, which have been shown to be in agreement with a broad range of empirical scaling relations, distribute energy and re-cycled ISM material over large scales and predict the existence of relatively "flat" and temporally invariant abundance gradients. Enhanced feedback schemes reduce significantly the scatter in the local stellar age-metallicity relation and, especially, the $[\mathrm{O} / \mathrm{Fe}]-[\mathrm{Fe} / \mathrm{H}]$ relation. The local $[\mathrm{O} / \mathrm{Fe}]$ distribution functions for our $\mathrm{L}^{*}$ discs show clear bimodality, with peaks at $[\mathrm{O} / \mathrm{Fe}]=-0.05$ and +0.05 (for stars with $[\mathrm{Fe} / \mathrm{H}]>-1$ ), consistent with our earlier work on dwarf discs.

Conclusions. Our results with "enhanced" feedback are inconsistent with our earlier generation of simulations realised with "conservative" feedback. We conclude that spatially-resolved metallicity distributions, particularly at high-redshift, offer a unique and under-utilised constraint on the uncertain nature of stellar feedback processes.
\end{abstract}

Key words. galaxies: abundances - galaxies: evolution - galaxies: formation - Galaxy: disk

\section{Introduction}

Radial abundance gradients and stellar age-metallicity relations provide two powerful constraints on the complex (and poorly understood) interplay between gas infall (e.g., cold flows from the intergalactic medium, coronal re-cycling of the underlying ISM), outflows (e.g., galactic fountains, superwinds, mass loading), stellar migration and radial gas flows, secular kinematic heating, interaction- and merger-driven energetics, and star formation efficiency, in driving the "inside-out" growth of disc galaxies. Local discs, including the Milky Way, provide one critical "boundary condition" for all models, in the sense that their present-day (i.e., gas-phase) radial metallicity gradients must be "negative" (i.e., decreasing in metallicity with increasing galactocentric radius) and of the order $-0.04 \mathrm{dex} / \mathrm{kpc}$.

Prior to 2011, no in situ measurements of abundance gradients at $z>0$ existed (particularly for "typical" star-forming and/or Grand Design spirals); three datasets have started to change this picture. MASSIV (Queyrel et al. 2012) found essentially flat gradients in a large number of discs (both isolated and interacting) at $z \sim 1$, although the challenging nature of this non-adaptive optics work makes the results particularly sensitive to spatial resolution limitations (Yuan et al. 2013). Conversely, Yuan et al. (2011) and Jones et al. (2013), using reconstructed source plane images of gravitationallylensed discs at $1.5<z<2.5$, found that in three out of their four systems, the inferred oxygen gradients were very steep $(-0.15 \rightarrow-0.3 \mathrm{dex} / \mathrm{kpc})$.

Complicating the picture further, one could try and make use of planetary nebulae sub-types within the Milky Way; as planetaries arise from stars ranging from $\sim 1 M_{\odot}$ to $\sim 8 M_{\odot}$, they provide something of a temporal probe of abundance gradients, albeit not measured in situ, but instead measured today at $z=0$, after experiencing several Gyrs or more of potential kinematic heating (both random "blurring" and systematic radial "migration"). Extant attempts to infer the temporal evolution of the Milky Way's gradient using such planetaries (Maciel et al. 2003; Stanghellini \& Haywood 2010) reflects that there remains a significant discrepancy between the claimed behaviour ${ }^{1}$. The Maciel et al. (2003) analysis results in an inferred gradient for the Milky Way which was steep at early times and flattened to today's value (-0.04 dex/kpc: Rupke et al. 2010). Conversely, the Stanghellini \& Haywood (2010) work leads to an inferred gradient at early times which is somewhat flatter than today's value (hence, a steepening with time).

Motivated by these empirical constraints, we examine here the role of energy feedback in shaping the temporal evolution of abundance gradients and age-metallicity relations within a

\footnotetext{
Very uncertain distance determinations and nebular emission approaches, presumably cloud the issue, but this is beyond the scope of our abilities to disentangle.
} 
sub-set of cosmological hydrodynamical disc simulations drawn from the MUGS (McMaster Unbiased Galaxy Simulations; Stinson et al. 2010) and MaGICC (Making Galaxies in a Cosmological Context; Brook et al. 2012b) suites. We will demonstrate how such observations can genuinely constrain the highly uncertain nature and magnitude of energy feedback underpinning galaxy formation.

In Pilkington et al. (2012a), we showed that "conventional" feedback schemes (i.e., those making use of $\sim 10-40 \%$ of the energy associated with each supernova (SN), to heat the surrounding ISM), when coupled with a classical SPH approach to hydrodynamics (independent of the SPH code employed), tended to result in galaxies with steep abundance gradients at redshifts $z>1(-0.15 \rightarrow-0.30 \mathrm{dex} / \mathrm{kpc}$ vs. $-0.04 \mathrm{dex} / \mathrm{kpc}$, today). Such "conventional" feedback schemes, when coupled to a grid-based approach to hydrodynamics at roughly the same resolution, also led to gradients steeper at high-redshift relative to the predicted present-day values $(-0.05 \rightarrow-0.10 \mathrm{dex} / \mathrm{kpc}$ vs. $-0.04 \mathrm{dex} / \mathrm{kpc}$, today), although systematically shallower than their SPH counterparts. This systematic difference was driven by the particular grid-based approach being adopted imposing a two-grid-cell minimum to the blastwave radius. We speculated then that any feedback scheme which distributed energy more efficiently on larger scales should result in flatter gradients ${ }^{2}$.

At the time of this earlier work (Pilkington et al. 2012a; Pilkington \& Gibson 2012), we did not have a clear manner in which to quantify the above inference. We are now in a position to show, in a direct manner, the impact on the temporal evolution of abundance gradients for $\mathrm{L}^{*}$ disc galaxies, when replacing the conventional feedback scheme employed for MUGS-g1536 and MUGS-g15785 with their now welltested MaGICC analogs. These enhanced feedback simulations (MaGICC-g1536 and MaGICC-g15784) were not available at the time of this previous work; combined with the aforementioned new empirical determinations of high-redshift in situ abundance gradients (most of which were also not available at the time of our initial study), this brief Research Note solidifies the more speculative conclusions we drew in (Pilkington et al. 2012a).

\section{Simulations}

We make use of two galaxies (g1536; g15784) drawn from the MUGS (Stinson et al. 2010) suite of $\mathrm{L}^{*}$ cosmological discs; these two systems are both isolated and experienced relatively quiescent assembly histories, since redshift $z \sim 2$. By avoiding strongly interacting major mergers and/or close pairs, the comparison between simulation and observation remains "like-withlike"; if we had not restricted ourselves to such isolated systems, we would necessarily have had to consider the impact that environment plays in flattening gradients during periods of strong interaction (e.g. Rupke et al. 2010; Few et al. 2012). Realised with the SPH code Gasoline (Wadsley et al. 2004), two variants of each galaxy were analysed - one using "conventional" feedback (MUGS) and one using "enhanced" feedback (MaGICC) ${ }^{3}$. Full

\footnotetext{
2 Turbulence driven by thermal instability can also be an efficient mechanism for mixing metals, as elucidated upon in the excellent work of Yang \& Krumholz (2012).

3 It should be emphasised that the assembly/merger histories for each MUGS-MaGICC "pair" (e.g., MUGS-g1536 and MaGICC-g1536) are identical; i.e., the differences discussed in Sects. 3 and 4 are due to internal (e.g., feedback, star formation, etc.), rather than external (e.g., merger history), processes.
}

details, including the methodology associated with star formation and feedback, for MUGS, can be found in Stinson et al. (2010) and Pilkington et al. (2012a). The MaGICC feedback prescription is outlined in a series of papers (Brook et al. 2011, 2012a,b,c; Pilkington et al. 2012b; Stinson et al. 2012, 2013).

Briefly, MUGS-g1536 and MUGS-g15784 employ a thermal feedback scheme in which $4 \times 10^{50} \mathrm{erg}$ per SN is made available to heat the surrounding interstellar medium (ISM), while their MaGICC analogs use $10^{51} \mathrm{erg} / \mathrm{SN}$. The MUGS (MaGICC) simulations were realised with a Kroupa et al. (1993) (Chabrier: Chabrier 2001) initial mass function (IMF) ${ }^{4}$. In the MaGICC runs, radiation energy feedback from massive stars is also included (in the $\sim 4 \mathrm{Myr}$ prior to the appearance of the first Type II SN from each star particle), albeit at an effective coupling efficiency $<1 \%$ (Brook et al. 2012b; Stinson et al. 2013). For both MUGS and MaGICC, cooling is disabled for gas particles situated within a blast region of size $\sim 100 \mathrm{pc}$, for a time period of $\sim 10$ Myr. Star formation is restricted to regions which are both sufficiently cool $(<10 \mathrm{kK})$ and dense (MUGS: $>1 \mathrm{~cm}^{-3}$; MaGICC: $>9 \mathrm{~cm}^{-3}$ ). Metal diffusion (Shen et al. 2010) is included in all runs.

To link the simulation nomenclature with their earlier appearances in the literature, MUGS-g1536 and MUGS-g15784 correspond to g1536 and g15784, respectively, in Stinson et al. (2010) and Pilkington et al. (2012a), while MaGICC-g1536 corresponds to the "Fiducial" run in Stinson et al. $(2013)^{5}$.

\section{Abundance gradients}

In Fig. $1^{6}$, we show the evolution of the gas-phase oxygen abundance gradients for both sets of realisations (g15784: + symbols; g1536: * symbols). Also included are the data sets of MASSIV (Queyrel et al. 2012), Yuan et al. (2011), and Jones et al. (2013). These are, to date, the only high redshift results against which our simulations can be compared. We also include results from studies of local planetary nebulae by Maciel et al. (2003, diamonds) and Stanghellini \& Haywood (2010, circles) ${ }^{7}$.

The lower ("conventional" feedback) pair of simulated galaxies show significant flattening from $z \sim 2$ to $z \sim 0$, as previously described by Pilkington et al. $(2012 a)^{8}$. Conversely, the upper pair show a dramatically different evolutionary sequence;

4 The MUGS runs assumed that the global metallicity $Z \equiv \mathrm{O}+\mathrm{Fe}$, while those of MaGICC assume $\mathrm{Z} \equiv \mathrm{O}+\mathrm{Fe}+\mathrm{C}+\mathrm{N}+\mathrm{Ne}+\mathrm{Mg}+\mathrm{Si}$; as such, the MUGS simulations underestimate the global metallicity by roughly a factor of two, and hence the impact of metallicity-dependent cooling (Pilkington et al. 2012a).

5 MaGICC-g1536 is also essentially the same as SG5LR, as reported in our earlier work (Brook et al. 2012b).

6 Our Fig. 1 here is a preferred update to Fig. 5 of Pilkington et al. (2012a).

7 It is important to note that the "high-redshift" abundance gradients inferred from sub-types of planetary nebulae today are strictly upper limits (as plotted), as any secular heating processes can really only flatten their in situ gradients to the values that we observe for them today; hence, the planetary nebulae data in Fig. 1 are drawn with downwardfacing arrows superimposed.

8 The $\sim 0.1 \rightarrow 0.3$ dex "deviations" in the flattening near redshift $z \sim 2$ are transient in nature, and due to periods of enhanced merger activity, as discussed by Pilkington et al. (2012a) and Few et al. (2012); this brief steepening of the gradient, followed by a subsequent "return" to the global flattening trend, is akin to the behaviour discussed eloquently by Rupke et al. (2010). Future work with a finer temporal output cadence will be required to better quantify the timescale upon which the gradient "returns" to its global flattening trend. 
B. K. Gibson et al.: Constraining sub-grid physics with high-redshift spatially-resolved metallicity distributions $(R N)$

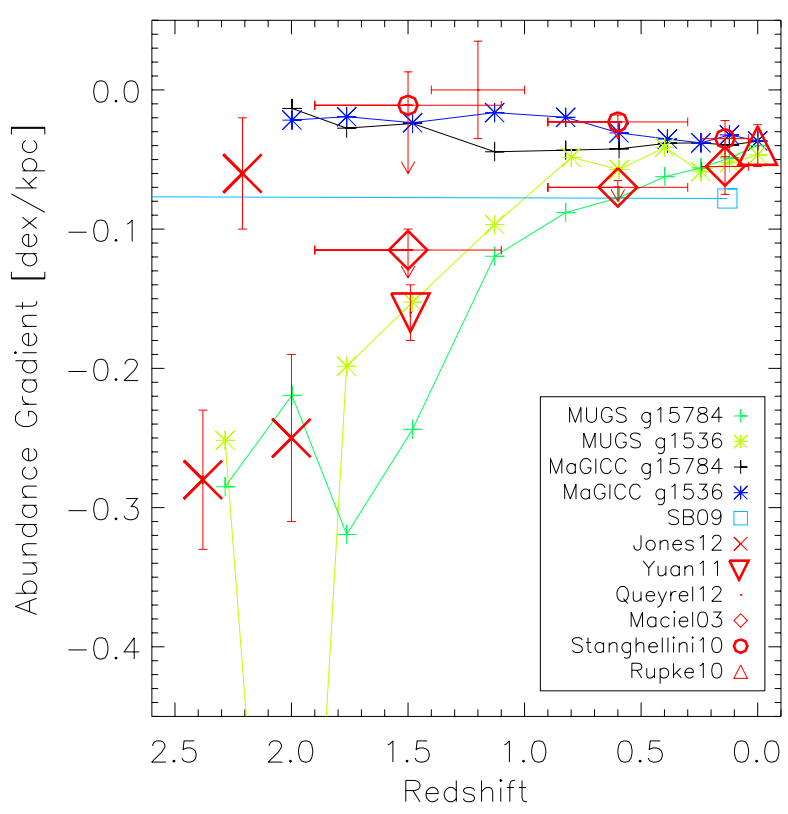

Fig. 1. Temporal evolution of the predicted oxygen abundance gradients associated with four simulated $\mathrm{L}^{*}$ disc galaxies - from the MUGS (Stinson et al. 2010) and MaGICC (Brook et al. 2012b) suites - in addition to an analytical model (SB09: Schönrich \& Binney 2009). Symbols correspond to empirical determinations of the abundance gradients in a sample of high-redshift lensed systems (Yuan et al. 2011; Jones et al. 2013), intermediate-redshift galaxies from MASSIV (Queyrel et al. 2012), local discs (Rupke et al. 2010), and the Milky Way (Maciel et al. 2003; Stanghellini \& Haywood 2010).

the stronger feedback implemented within the MaGICC scheme, results in essentially flat gradients at high-redshift, with minimal steepening (rather than flattening) with time. As the MaGICC feedback scheme re-distributes energy and re-cycled ISM material over much greater galactic scales (via winds driving lowangular momentum inner disc and bulge gas to the corona, where it then cools, falls back preferentailly to the outer disc, and re-enters the star-forming region preferentially as an inplane radial "flow"; the re-cycling pattern is described in more detail by Brook et al. 2011, 2012a) such flat (and essentially temporally-invariant) gradients are consistent with our interpretation of the difference between the grid and particle-based simulations, as noted above, and in our earlier work (Pilkington et al. 2012a). Specifically, the flatter gradients seen in the MaGICC scheme at $z>0$ are due to the combined effect of (a) "metal re-cycling" via outflows (which re-distributes metals), and (b) "ISM re-structuring" via outflows (which re-distributes the ISM and hence radial star formation profile).

It is worth reminding the reader as to the predicted temporal evolution of the gradients from classical "analytical" chemical evolution models for the Milky Way (Chiappini et al. 2001; Mollá \& Díaz 2005; Schönrich \& Binney 2009). As reported in Pilkington et al. (2012a), the models of Chiappini et al. (2001) and Mollá \& Díaz (2005) show behaviour which is indistinguishable from that seen in the stronger feedback MaGICC-g1536 and MaGICC-g15784 models. The model of Schönrich \& Binney (2009) is similar, in the sense of showing very little temporal evolution, albeit it remains somewhat steep at all times.

It is worth asking how the transition from "conventional" MUGS feedback to stronger MaGICC feedback impacts on the inferred gradient in $[\mathrm{O} / \mathrm{Fe}]$. Having addressed the former (Pilkington \& Gibson 2012), albeit briefly, we show in Fig. 2 the mass-weighted stellar $[\mathrm{O} / \mathrm{Fe}]$ gradients at redshift $z=0$ from

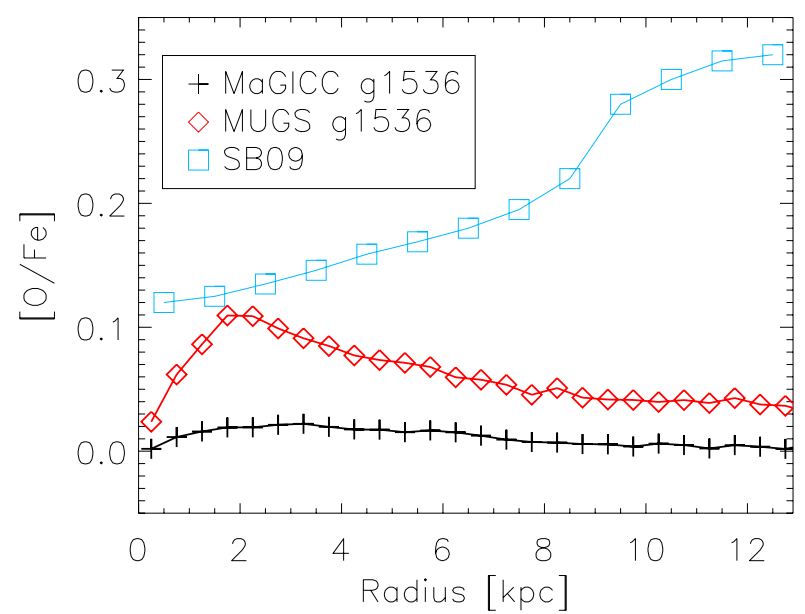

Fig. 2. Predicted present-day, stellar mass-weighted, radial $[\mathrm{O} / \mathrm{Fe}]$ gradients for the MaGICC (Brook et al. 2012b) and MUGS (Stinson et al. 2010) realisations of simulation g1536, compared with that predicted by an analytical model of the Milky Way (SB09: Schönrich \& Binney 2009).

MUGS-g1536, MaGICC-g1536, and the analytical model of Schönrich \& Binney (2009). Both the MUGS and MaGICC realisations possess very flat gradients $(<0.005 \mathrm{dex} / \mathrm{kpc})$, similar to those observed by Sánchez-Blázquez et al. (2009), although the uncertainties associated with inferring mass- or light-weighted $[\alpha / \mathrm{Fe}]$ gradients from integrated spectroscopy of face-on discs can be significant (see also Fenner et al. 2006). In contrast with the MaGICC predictions, the analytical models of Schönrich \& Binney (2009) predict positive integrated light/mass gradients in $[\mathrm{O} / \mathrm{Fe}]$, within the star forming part of the disc, on the order of $\sim+0.02 \mathrm{dex} / \mathrm{kpc}$.

A characteristic of the model of Schönrich \& Binney (2009), relative to several classical models of galactic chemical evolution (Chiappini et al. 2001; Mollá \& Díaz 2005), is the inclusion of radial gas flows 9 . Such flows are also a natural outcome of our enhanced MaGICC feedback scheme (Brook et al. 2011). While it can be challenging to infer the signal of $\sim 1-2 \mathrm{~km} \mathrm{~s}^{-1}$ flows, when superimposed upon a (say) $\sigma_{r} \sim 30 \mathrm{~km} \mathrm{~s}^{-1}$ velocity dispersion profile (both within the simulations and (especially) in nature (Dame 1993)), we have attempted to do so. Formally, for MUGS-g1536 (at redshift $z=0$ ), we find inward radial (cold) gas flows of $\sim 3 \mathrm{~km} \mathrm{~s}^{-1}\left(\sim 1 \mathrm{~km} \mathrm{~s}^{-1}\right)$ within a $\pm 2 \mathrm{kpc}$ thick annulus at $13<r<17 \mathrm{kpc}(7<r<8 \mathrm{kpc})$; for MaGICC-g1536, the radial flows (again, at $z=0$ ) are much larger: $\sim 12 \mathrm{~km} \mathrm{~s}^{-1}$ $\left(\sim 8 \mathrm{~km} \mathrm{~s}^{-1}\right)$ at the same galactocentric radii. A more detailed analysis of the temporal evolution of the gas flows will be required to disentangle the relative roles, within MaGICC, of recycling of the ISM over increasingly large galactic scales and the increasingly more substantial radial gas flows.

\section{Age metallicity relations}

In Fig. 3, for MaGICC-g1536 (left column) and MUGS-g1536 (right column), we show their inferred local "solar neighbourhoods" stellar metallicity distribution function (MDF: top row), age-metallicity relation (AMR: second row), $[\mathrm{O} / \mathrm{Fe}]-[\mathrm{Fe} / \mathrm{H}]$ distribution (third row), and $[\mathrm{O} / \mathrm{Fe}]$ distribution function (bottom row). The solar neighbourhood is taken as the region $3<$ $r_{\mathrm{d}}<3.5$, where $r_{\mathrm{d}}$ is the radius in units of disc scalelength. While

\footnotetext{
9 See also the radial flow model of Spitoni et al. (2013), for insightful commentary on the issue of radial flows in disc galaxies.
} 

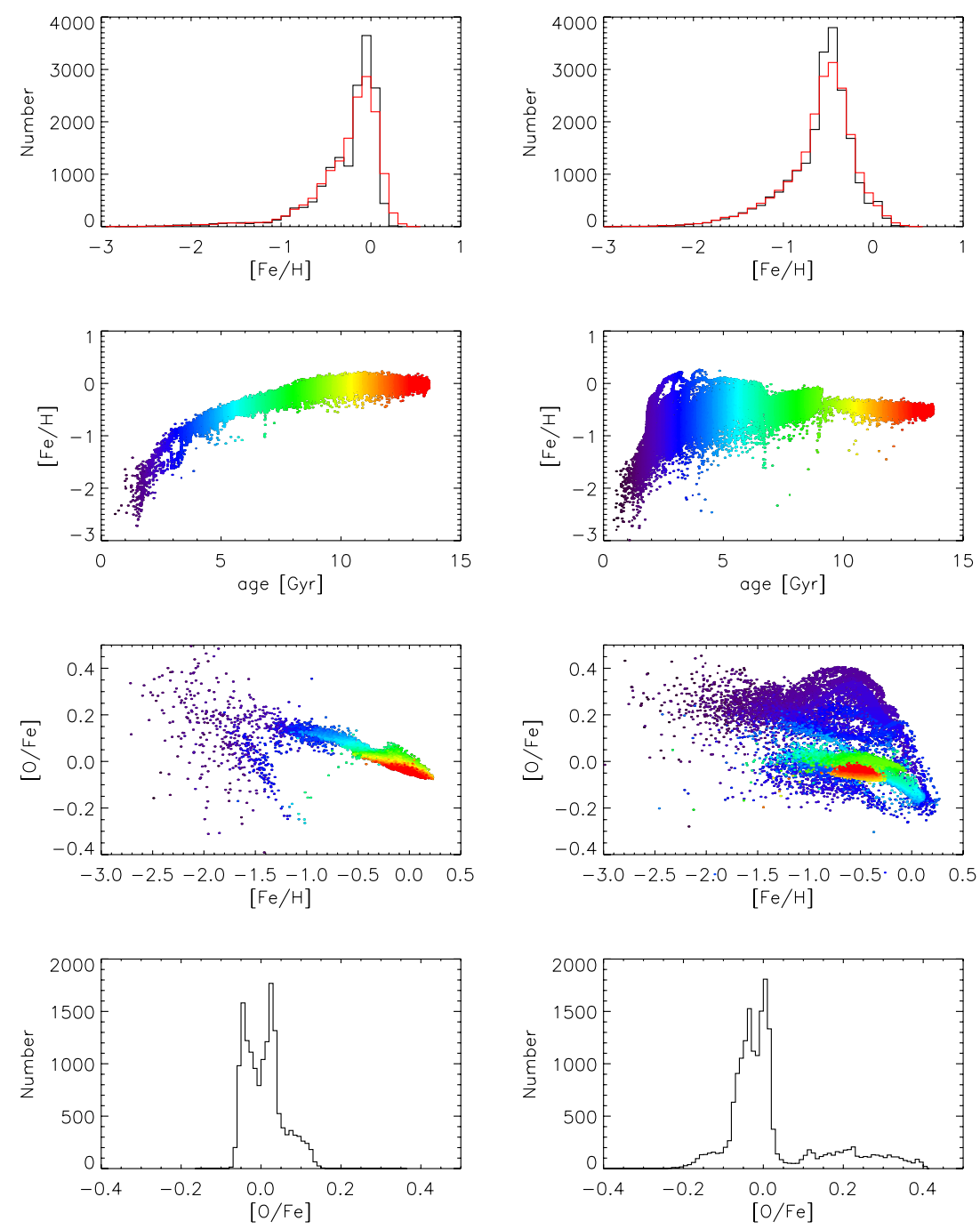

Fig. 3. Predicted metallicity distribution functions (top row); age-metallicity relations (second row); $[\mathrm{O} / \mathrm{Fe}]-[\mathrm{Fe} / \mathrm{H}]$ relations (third); and $[\mathrm{O} / \mathrm{Fe}]$ distributions (bottom row), for "solar neighbourhood" regions of the MaGICC (left column: Stinson et al. 2013) and MUGS (right column: Stinson et al. 2010) realisations of simulation g1536. the impact of the enhanced feedback associated with MaGICC is readily apparent in the AMR and $[\mathrm{O} / \mathrm{Fe}]-[\mathrm{Fe} / \mathrm{H}]$ planes, the effect is more subtle in the MDF and $[\mathrm{O} / \mathrm{Fe}]$ distribution function. The remarkably tight (effectively temporally invariant scatter) and correlated AMRs in the (analogous) simulated solar neighbourhood, for this $\mathrm{L}^{*}$ realisation, is similar to that encountered in our earlier work on dwarf discs (Pilkington et al. 2012b). At a given $[\mathrm{Fe} / \mathrm{H}]$, within the solar neighbourhood, two (roughly) parallel loci in $[\mathrm{O} / \mathrm{Fe}]$ co-exist (for $[\mathrm{Fe} / \mathrm{H}]>-1$ ) with a separation of $\sim 0.1$ dex (bottom left panel of Fig. 3); this separation is, admittedly, smaller than that seen in the solar neighbourhood of the Milky Way ( $\sim .3$ dex: e.g., Fig. 3 of Fuhrmann \& Bernkopf 2008), although in a qualitative sense the behaviour is not dissimilar. An earlier detailed examination of the origin of these offset sequences (in a dwarf disc realisation) demonstrated significant parallels with said empirical sequences (drawing links with "thin" and "thick" discs, and highlighting the role of radial migration - Brook et al. 2012c); such behaviour, including offset loci in $[\mathrm{O} / \mathrm{Fe}]$ for $[\mathrm{Fe} / \mathrm{H}]>-1$ is reflected in the $\mathrm{L}^{*}$ realisations described here (see the bottom row of Fig. 3, where the bimodal $[\mathrm{O} / \mathrm{Fe}]$ distributions associated with $[\mathrm{Fe} / \mathrm{H}]>-1$ stars in the simulated solar neighbourhoods is apparent.

\section{Conclusions}

Negative abundance gradients at redshift zero provide a local boundary condition for galaxy evolution models, in support of inside-out disc growth. Importantly, we are seeing the beginnings of associated boundary conditions on the temporal evolution of said metallicity gradients, with recent in situ determinations of radial abundance gradients in typical star-forming and/or Grand Design spirals at redshifts $2.4<z<1.0$ (e.g. Yuan et al. 2011; Queyrel et al. 2012; Jones et al. 2013). These developments are very much on the leading-edge of what can be done today; hence, the still contradictory nature of the results - i.e., whether or not abundance gradients steepen or flatten with time - should not be surprising. Time (and additional high-redshift data) will certainly settle this observational issue; despite this, the power to use these in situ observations is enticing, and has led us to drive this effort to use their results to constrain the very uncertain nature of sub-grid physical energy feedback within models of galaxy evolution.

To this end, we have analysed a suite of simulated $\mathrm{L}^{*}$ discs, realised with different feedback implementations. The enhanced feedback models of the MaGICC programme (Brook et al. 2012b; Stinson et al. 2012), which have been shown to be in agreement with a broad range of present-day empirical scaling relations, predict that gradients should only mildly steepen with time. These relatively "flat" and temporally invariant abundance gradients result from feedback which distributes energy and recycled ISM material over large scales, coupled with stronger radial gas flows. These results are consistent with extant analytical models of galactic chemical evolution, the inferred Milky Way gradient at high-redshift by Stanghellini \& Haywood (2010), and 
in situ abundance gradients at high-redshift, as determined by Queyrel et al. (2012). By contrast, the simulations which incorporated relatively weak feedback, without including early stellar feedback from massive stars prior to exploding as SNs, results in metallicity gradients that are steep at high redshift (consistent with in situ abundance gradients at high-redshift determined by Yuan et al. 2011 and Jones et al. 2013, and the inferred Milky Way gradient at high-redshift by Maciel et al. 2003). We do not wish to leave the reader with any notions regarding the validity of MaGICC or MUGS feedback schemes based upon the in situ determination of abundance gradients at high-redshift; we are not in a position to do so yet. What is true though is that such empirical determinations possess a unique ability to constrain the uncertain nature of sub-grid feedback within galaxy-scale hydrodynamical simulations.

Enhanced feedback also results in significantly reduced scatter in the local stellar age-metallicity relation and, especially, the $[\mathrm{O} / \mathrm{Fe}]-[\mathrm{Fe} / \mathrm{H}]$ relation. The local $[\mathrm{O} / \mathrm{Fe}]$ distribution functions for our $\mathrm{L}^{*}$ discs show clear bimodality, with peaks at $[\mathrm{O} / \mathrm{Fe}]=-0.05$ and +0.05 (for stars with $[\mathrm{Fe} / \mathrm{H}]>-1$ ); as noted in Sect. 4, such a separation is qualitatively (if not quantitatively) similar to that seen in the solar neighbourhood of the Milky Way. A detailed analysis of both the age-metallicity relations and metallicity distribution functions associated with these simulations will form the basis of a future study.

In light of the success of the MaGICC formulation for feedback at redshift zero (Brook et al. 2012a,b,c; Pilkington \& Gibson 2012; Pilkington et al. 2012b; Stinson et al. 2012, 2013), it may be tempting emphasise the consistency between classical models of chemical evolution, MaGICC-g1536, and MaGICCg15784, with the empirical data of Queyrel et al. (2012) and the planetary nebula work of Stanghellini \& Haywood (2010). Having said that, there are no a priori reasons to doubt the gradients inferred from lensed discs at high-redshift (Yuan et al. 2011, 2013; Jones et al. 2013), nor to dismiss the planetary nebulae work of Maciel et al. (2003). Future observational campaigns, and detailed intercomparisons of the disparate planetary nebulae samples, will surely provide definitive and pivotal conclusions as to whether the somewhat flat and temporally invariant gradients predicted with the MaGICC feedback formulation stand the test of time, or whether the situation is more complicated (or at least varied) than we envision. Such observations are a unique and, until now, missing constraint/boundary condition on models of galaxy evolution.

Acknowledgements. B.K.G. acknowledges the support of the UK's Science \& Technology Facilities Council (ST/J001341/1). K.P. acknowledges the support of STFC through its PhD Studentship programme (ST/F007701/1). The generous allocation of resources from STFC's DiRAC Facility (COSMOS: Galactic Archaeology) is gratefully acknowledged. We also thank the DEISA consortium, co-funded through EU FP6 project RI-031513 and the FP7 project RI-222919, for support within the DEISA Extreme Computing Initiative, and the University of Central Lancashire's High Performance Computing Facility.

\section{References}

Brook, C. B., Governato, F., Roškar, R., et al. 2011, MNRAS, 415, 1051 Brook, C. B., Stinson, G., Gibson, B. K., et al. 2012a, MNRAS, 419, 771 Brook, C. B., Stinson, G., Gibson, B. K., Wadsley, J., \& Quinn, T. 2012b, MNRAS, 424, 1275

Brook, C. B., Stinson, G. S., Gibson, B. K., et al. 2012c, MNRAS, 426, 690 Chabrier, G. 2001, ApJ, 554, 1274

Chiappini, C., Matteucci, F., \& Romano, D. 2001, ApJ, 554, 1044

Dame, T. M. 1993, in Back to the Galaxy, eds. S. S. Holt, \& F. Verter, AIP Conf. Ser., 278, 267

Fenner, Y., Gibson, B. K., Gallino, R., \& Lugaro, M. 2006, ApJ, 646, 184

Few, C. G., Gibson, B. K., Courty, S., et al. 2012, A\&A, 547, A63

Fuhrmann, K., \& Bernkopf, J. 2008, MNRAS, 384, 1563

Jones, T., Ellis, R. S., Richard, J., \& Jullo, E. 2013, ApJ, 765, 48

Kroupa, P., Tout, C. A., \& Gilmore, G. 1993, MNRAS, 262, 545

Maciel, W. J., Costa, R. D. D., \& Uchida, M. M. M. 2003, A\&A, 397, 667

Mollá, M., \& Díaz, A. I. 2005, MNRAS, 358, 521

Pilkington, K., \& Gibson, B. K. 2012, in Galactic Archaeology: Near-Field Cosmology and the Formation of the Milky Way, eds. W. Aoki, M. Ishigaki, T. Suda, T. Tsujimoto, \& N. Arimoto, ASP Conf. Ser., 458, 241

Pilkington, K., Few, C. G., Gibson, B. K., et al. 2012a, A\&A, 540, A56

Pilkington, K., Gibson, B. K., Brook, C. B., et al. 2012b, MNRAS, 425, 969

Queyrel, J., Contini, T., Kissler-Patig, M., et al. 2012, A\&A, 539, A93

Rupke, D. S. N., Kewley, L. J., \& Barnes, J. E. 2010, ApJ, 710, L156

Sánchez-Blázquez, P., Courty, S., Gibson, B. K., \& Brook, C. B. 2009, MNRAS, 398,591

Schönrich, R., \& Binney, J. 2009, MNRAS, 396, 203

Shen, S., Wadsley, J., \& Stinson, G. 2010, MNRAS, 407, 1581

Spitoni, E., Matteucci, F., \& Marcon-Uchida, M. M. 2013, A\&A, 551, A123

Stanghellini, L., \& Haywood, M. 2010, ApJ, 714, 1096

Stinson, G. S., Bailin, J., Couchman, H., et al. 2010, MNRAS, 408, 812

Stinson, G. S., Brook, C., Prochaska, J. X., et al. 2012, MNRAS, 425, 1270

Stinson, G. S., Brook, C., Macciò, A. V., et al. 2013, MNRAS, 428, 129

Wadsley, J. W., Stadel, J., \& Quinn, T. 2004, New Astron., 9, 137

Yang, C.-C., \& Krumholz, M. 2012, ApJ, 758, 48

Yuan, T.-T., Kewley, L. J., Swinbank, A. M., Richard, J., \& Livermore, R. C. 2011, ApJ, 732, L14

Yuan, T.-T., Kewley, L. J., \& Rich, J. 2013, ApJ, 767, 106 